used their approach to fabricate a 12-channel vertical-cavity surface-emitting laser array transmitter; tests show that data transmission at rates of up to $12.5 \mathrm{Gbit} \mathrm{s}^{-1}$ per channel is possible with their design.

\section{SPECTRAL FILTERS}

\section{Atomic frequency comb}

Appl. Phys. Lett. 101, 191112 (2012)

Ultrasound-modulated optical tomography is showing promise for the highly sensitive, deeply penetrating imaging of soft tissue. However, the sensitive detection of ultrasound from biological samples requires strong spectral filters that can discriminate between information in the carrier frequency and sideband frequencies of the modulated light. David McAuslan and co-workers from the University of Otago in New Zealand have now used quantum memory techniques to achieve extremely sensitive ultrasound detection. The researchers used a $\operatorname{Pr}^{3+}: \mathrm{Y}_{2} \mathrm{SiO}_{5}$ crystal as the quantum memory and generated two atomic frequency combs at $\pm 1.5 \mathrm{MHz}$ to store the ultrasound. They separated the combs by employing a transmission window that was fixed to the frequency of the input laser, and used a $606 \mathrm{~nm}$ laser beam that was phasemodulated by an acousto-optic modulator. This stored the resulting sidebands in the atomic frequency comb, resulting in the formation of an echo $6.667 \mathrm{~s}$ after the input pulse beam was transmitted. For a $1 \mathrm{~mW}$ input pulse beam, the peak level of noise in the detection window was $49 \mathrm{~dB}$ below the level of the input pulse beam, which is a significant improvement over other ultrasound detection methods based on rare-earthdoped crystals.

\section{NONLINEAR DYNAMICS}

\section{Quantum chaos}

Nature Commun. 3, 2214 (2012)

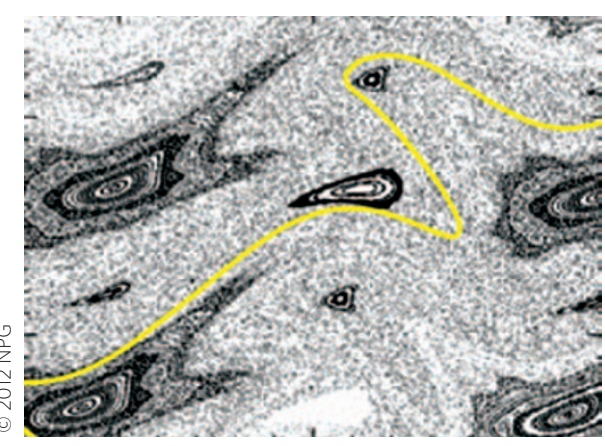

There is particular interest in systems whose dynamical behaviour can be driven from regular to chaotic by a small perturbation. A number of intriguing quantum-dynamical features have been unveiled so far, but there is still much to be learned about the transition to chaos when it occurs abruptly at critical values. Now, Gabriela Lemos and co-workers from Universidade Federal do Rio de Janeiro in Brazil have proposed a scheme for observing the transition by making a direct measurement of the optical Wigner distribution. The researchers fired the beam from a He-Ne laser into a quantum-kicked harmonic oscillator, where it reflected multiple times between a spatial light modulator and a mirror. The spatial light modulator produced an instantaneous kick perturbation and the harmonic evolution relevant to the effective Planck constant was implemented by sections of free propagation and a cylindrical lens. The displacement and tilting of a steering mirror at the entrance of a three-mirror Sagnac interferometer displaced the optical field by an amount $Q$ and changed its direction of propagation by an amount $P$. The researchers obtained Wigner distributions corresponding to the $Q-P$ map by an interferometric method. The results showed the transition from regular to chaotic dynamics, in which the parameters of the kick strength and effective Planck constant were tuned.

\section{QUANTUM OPTICS}

\section{Tripartite entanglement \\ Nature Phys. 9, 19-22 (2012)}

The generation of correlated particles and a means of assessing their states are both essential techniques for investigating quantum mechanical phenomena. Although researchers have entangled discrete variables of up to 14 ions and continuous variables between three intense optical beams, entangling the continuous properties of three or more individual particles remains a challenge. Now, Krister Shalm and co-workers from the University of Waterloo and the University of Calgary in Canada have not only generated tripartite continuousvariable entanglement between three separate particles, but also proposed a scheme to verify the correlated state. The researchers generated three entangled photons from two periodically poled potassium titanyl phosphate crystals by cascaded spontaneous parametric downconversion. First, they used a $404 \mathrm{~nm}$ pump laser to generate a pair of daughter photons at $776 \mathrm{~nm}$ and $842 \mathrm{~nm}$. They then used the photon at $776 \mathrm{~nm}$ to generate a pair of granddaughter photons at $1,530 \mathrm{~nm}$ and $1,570 \mathrm{~nm}$. To verify the correlated states of the photons, the researchers used a continuous-variable entanglement criterion; instead of measuring the frequencies of each individual photon, they measured the frequency of the pump, thus providing a direct measurement of the total frequency of the three daughter photons required by the inequalities. By putting the uncertainty in the arrival times between two of the three photons into the inequalities, they verified that the three photons strongly violated inequalities and were therefore tripartite continuous-variable entangled. $\mathrm{NH}$

\section{SIGNAL PROCESSING}

\section{Cavity-less source \\ Opt. Express 20, B419-B427 (2012)}

Researchers from the University of California in the USA have demonstrated the benefits of employing a high-fidelity cavity-less optical pulse source in an analog-to-digital signal conversion scheme. Their source generates optical pulses in a single pass by using a series of cascaded phase modulators to chirp the light from a continuous-wave $100 \mathrm{~mW}$ distributed feedback laser. The chirped light is then compressed into short pulses using a section of dispersion-compensating fibre. They used the resulting 3 ps optical pulses, which had a repetition rate of $2 \mathrm{GHz}$ and a signal-to-noise ratio of over $40 \mathrm{~dB}$, in an analog-to-digital sampling pre-processor, with 8.2 and 7.0 effective bits for analog input signals at frequencies of $0.2 \mathrm{GHz}$ and $40 \mathrm{GHz}$, respectively. The researchers say their demonstration shows that cavity-less pulse sources are a viable alternative to mode-locked lasers in signal processing applications. $\quad O G$

\section{IMAGING}

\section{Ultrafast atomic probe}

Phys. Rev. Lett. 109, 233002 (2012)

Researchers from the USA and China have studied the characteristics of laser-induced electron diffraction that make it suitable for use as an ultrafast atomic probe. By exploiting the strong wavelength dependence of an intense laser-atom interaction, Junliang $\mathrm{Xu}$ and colleagues report high-resolution photoelectron momentum distributions of rare-gas atoms recorded at mid-infrared wavelengths $(>100 \mu \mathrm{m})$. Mid-infrared lasers provide large-range momentum transfer, which is critical for achieving good spatial resolution for molecular imaging. The researchers have shown that the strong shortrange atomic potential dominates the electronion interaction while the valence electrons remain transparent, which is a prerequisite for imaging. For recollision energies of more than $100 \mathrm{eV}$, they found that the electron-ion differential cross-sections of rare gas atoms are nearly the same for neutral atoms and singly charged ions, and that they are independent of the laser parameters.

Written by Oliver Graydon, Noriaki Horiuchi, David Pile and Rachel Won. 